\title{
Accurate and Efficient Measurement of Impurity (Dilute) Diffusion Coefficients without Isotope Tracer Experiments
}

\author{
Qiaofu Zhang, Zhangqi Chen, Wei Zhong, Ji-Cheng Zhao* \\ Department of Materials Science and Engineering, The Ohio State University, Columbus, OH, 43210, USA \\ * Corresponding author. Tel.: +1 614292 9462. E-mail address: zhao.199@ osu.edu (J.-C. Zhao)
}

\begin{abstract}
Most impurity diffusion coefficients were measured in the 1960s and 1970s using tracer experiments with only 14 sets of tracer measurements performed worldwide in the past 15 years. It is thus impractical to rely on tracer experiments to obtain the missing thousands of impurity diffusion coefficients. A recently developed forward-simulation analysis can change the scenario by reliably extracting impurity diffusion coefficients from regular diffusion couple profiles. A comparison of 52 values obtained from the forward-simulation analysis with tracer experiment values in the literature shows an excellent agreement, thus validating the reliability of the forward-simulation analysis in obtaining accurate impurity diffusion coefficients.
\end{abstract}

Keywords: Impurity diffusion coefficient, Dilute diffusion coefficient, Isotope tracer experiment, Forward-simulation analysis, Diffusion multiples

Diffusion plays an essential role in key materials processes such as solidification and casting, sintering, homogenization, precipitation (growth and coarsening), welding and joining, surface modification, and creep deformation. Therefore, diffusion coefficients are among the most fundamental materials properties. It is the self-diffusion coefficients and impurity (dilute) diffusion coefficients together that lay the foundation for reliable diffusion (mobility) databases that are essential for MGI/ICME-based materials design and process optimization (MGI refers to the Materials Genome Initiative [1] and ICME stands for the Integrated Computational Materials Engineering [2,3]). In other words, the self-diffusion and impurity diffusion coefficients are the foundation of diffusion (mobility) databases [4] in a similar way as the lattice stabilities [5,6] are the foundation of thermodynamic databases.

Both self-diffusion and impurity diffusion coefficients are predominately measured using radioactive isotope tracer diffusion experiments [7,8] which usually produce very reliable datasets but are laborious and expensive to perform. Through audacious work of many researchers over the past seven decades, the self-diffusion coefficients of 42 out of the 61 non-radioactive solid metals in the periodic table are now available (See the Supplementary Information). The number for the impurity diffusion coefficients is far more daunting since each of the 61 metallic elements can be a matrix while the solute can be one of the other 60 metals, $\mathrm{Hg}$, the 6 metalloids (B, Si, Ge, As, Sb and Te), or the 4 solid nonmetals (C, P, S and Se). Thus, the total number of impurity diffusion coefficient sets could be as many as 4331 which have not 
even included the gaseous elements as solutes or all the Group 7 elements that are either a liquid at ambient temperature or too radioactive to handle without elaborate protections. By carefully counting through the comprehensive collection (and assessment) of Neumann and Tuijn [9] that has included data up until 2007, we found a total of 374 sets of impurity diffusion coefficients in 44 metal matrixes (A set of impurity diffusion coefficients here refers to the results of a study of one solute in one element matrix at three or more temperatures such that an Arrhenius equation of the impurity diffusion coefficient can be defined.) A search of the literature from 2007 to August 2016 yielded only 3 additional sets of tracer experiments on pure metals [10,11]. It is thus clear that less than $10 \%$ of the required impurity diffusion coefficients have been determined experimentally.

Even though significant progress has been made in recent years in computing the impurity diffusion coefficients using density functional theory (DFT) based methods [12,13,14], accurate experimental measurements are still essential for the establishment of reliable diffusion (mobility) databases before the computed values are fully validated for accuracy.

Figure 1 clearly shows that most tracer experiments on impurity diffusion coefficients in pure metals were performed back in the 1960s and the 1970s with dwindling numbers in the 1980s and the 1990s. Since the year 2000, only 14 sets of tracer experiments on impurity diffusion coefficients were found/reported (averaged to only $\sim 1$ set/year!). Such a low number of tracer experiments performed worldwide in the recent decade makes it impractical to rely on the tracer experiments to obtain the missing impurity diffusion coefficients $(4331-377=3954$ sets $)$; thus, alternative, non-tracer, methods are highly sought after to accurately and effectively measure the impurity diffusion coefficients.

While isotope tracer experiments are no longer been widely performed, regular diffusion couple experiments are still being extensively performed worldwide to measure composition-dependent interdiffusion coefficients (also called chemical diffusion coefficients) and phase diagrams. It would be highly desirable to obtain accurate impurity diffusion coefficients from the regular diffusion couple experiments. As a matter of fact, the so-called incremental diffusion couple that is made up of a very dilute alloy with a pure element has been employed to obtain impurity diffusion coefficients $[15,16]$, assuming the interdiffusion coefficients obtained from such a diffusion couple is composition independent and equals to the impurity diffusion coefficients. With the same assumptions, thin-film diffusion couples [17] were also used to measure impurity diffusion coefficients. Individual dilute alloys or thin film samples need to be made for such experiments; and for this reason, the methods are not widely employed. It would be more cost-effective and convenient to extract impurity diffusion coefficients directly from regular diffusion couples such as pure metal to pure metal diffusion couples, considering the large number of impurity diffusion coefficients that still need to be determined.

The Boltzmann-Matano analysis [18] is usually performed to evaluate the interdiffusion coefficients $\widetilde{D}$ at a composition $c^{\prime}$ from a concentration profile $C(x, t)$ obtained from a diffusion couple:

$$
\widetilde{D}=-\frac{1}{2 t}\left[\frac{\partial C}{\partial x}\right]^{-1} \int_{C_{L}}^{C^{\prime}}\left(x-x_{M}\right) d C
$$

where $x$ is the position, $t$ is the diffusion time, $C_{L}$ is the left-end concentration, and $x_{M}$ is the position of the Matano plane that is determined by mass balance: 


$$
\int_{-\infty}^{x_{M}}\left[C(x)-C_{L}\right] d x+\int_{x_{M}}^{+\infty}\left[C(x)-C_{R}\right] d x=0
$$

where $C_{R}$ is the right-end concentration of the diffusion couple. A concentration ratio $Y(x)=\frac{C(x)-C_{L}}{C_{R}-C_{L}}$ was introduced by Sauer and Freise [19] to avoid the need to compute the Matano plane position such that:

$$
\widetilde{D}=-\frac{1}{2 t}\left[\frac{d Y}{d x}\right]_{x^{\prime}}^{-1}\left(\left[1-Y\left(x^{\prime}\right)\right] \int_{-\infty}^{x^{\prime}} Y(x) d x+Y\left(x^{\prime}\right) \int_{x^{\prime}}^{+\infty}[1-Y(x)] d x\right)
$$

Nevertheless, the Sauer-Freise method still depends on the concentration gradient of the diffusion profile to evaluate the interdiffusion coefficients, similar to the Boltzmann-Matano analysis. There is uncertainty in determining the concentration gradient at the two ends of a diffusion couple profile when the noise/variation in the electron-probe microanalysis (EPMA) measurement is of the same order of magnitude of the very dilute concentrations, which renders both the Boltzmann-Matano and the SauerFreise methods incapable of accurately evaluating the impurity diffusion coefficients.

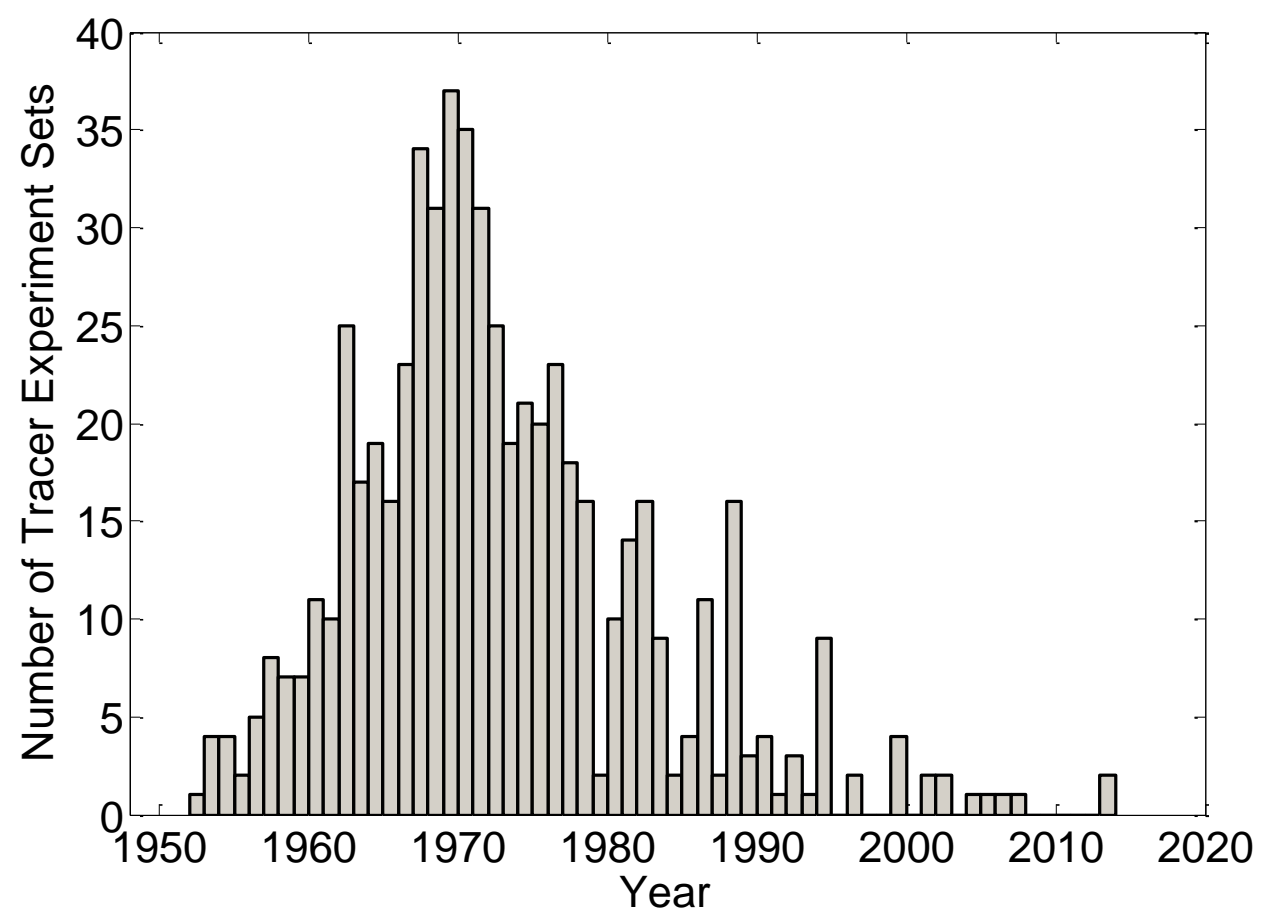

Fig. 1. Histogram for the number of isotope tracer experiments performed/published worldwide to obtain impurity (dilute) diffusion coefficients in pure metals. A set of data here refers to the results of a study of one solute in one element matrix at three or more temperatures such that an Arrhenius equation of the impurity diffusion coefficient can be defined. The data from the 1950s to 2007 are counted from the compilation of Neumann and Tuijn [9] and the data after 2007 are based on a recent literature search by the current authors.

Hall [20] devised a method to extract impurity diffusion coefficients from regular diffusion couple profiles by assuming that the interdiffusion coefficients near the pure metal ends of a diffusion couple are constants at arbitrarily defined small composition regions near the pure metals. He then obtain the 
interdiffusion coefficients by analyzing the profile of $u$ against $\lambda$ with a linear fit $u=\mathrm{h} \lambda+\mathrm{k}$. Here $u$ and $\lambda$ are defined from: $Y(x)=\frac{C(x)-C_{L}}{C_{R}-C_{L}}=\frac{1}{2}[1+\operatorname{erf}(u)]$ and $\lambda=x / \sqrt{t}$ ( $x$ is distance and $t$ is time), and $\mathrm{h}$ and $\mathrm{k}$ are fitting parameters from the experimental concentration profiles in the small composition regions near the pure elements. The interdiffusion coefficients (assuming to be equal to the impurity diffusion coefficients at the very dilute concentrations) at a concentration $C^{\prime}$ near the left composition $C_{L}$ and right composition $C_{R}$ can be estimated by equations

$$
\tilde{D}\left(C^{\prime}\right)=\frac{1}{4 h_{1}^{2}}\left[1+\frac{2 k_{1}}{\sqrt{\pi}} \exp \left(u^{2}\right) \times Y\left(C^{\prime}\right)\right] \quad \text { and } \quad \tilde{D}\left(C^{\prime}\right)=\frac{1}{4 h_{2}^{2}}\left\{1-\frac{2 k_{2}}{\sqrt{\pi}} \exp \left(u^{2}\right)\left[1-Y\left(C^{\prime}\right)\right]\right\}
$$

respectively, with the evaluated linear fitting parameters $h_{1}, k_{1}$ for left side and $h_{2}, k_{2}$ for the right side of the diffusion couple. The Hall method works when the interdiffusion coefficients are near constant at both ends of a diffusion couple. For systems with strong composition-dependent diffusion coefficients, the Hall method breaks down. In addition, the selection of the small composition regions to apply the Hall analysis is quite arbitrary and the obtained impurity diffusion coefficients sometimes are not on the extrapolated lines of the interdiffusion coefficients, creating a dilemma on deciding which sets of (impurity or interdiffusion) data to trust.

A forward-simulation analysis was developed by Zhang and Zhao [21,22] to reliably evaluate both interdiffusion and impurity diffusion coefficients from regular (include pure metal to pure metal) diffusion couple profiles. Instead of using the traditional methods based on concentration gradients to evaluate diffusion coefficients at many points across a concentration profile, the forward-simulation analysis evaluates the interdiffusion coefficient $\widetilde{D}\left(X_{B}\right)\left(X_{B}\right.$ is the mole fraction of element B) by:

1) Performing a smoothing of the raw experimental concentration profile data to reduce noises introduced by the EPMA measurement (Only the smooth composition profiles $C_{\text {expt }}(x)$ are used for the diffusion coefficient analysis (where $x$ is distance));

2) Employing the Sauer-Freise method to extract the composition-dependent interdiffusion coefficients $D_{S-F}\left(X_{B}\right)$ which provide the first-guess diffusion coefficients function: $\widetilde{D}\left(X_{B}\right)=$ $\exp \left(f\left(X_{B}\right)\right)$ while $f\left(X_{B}\right)$ is usually a polynomial function or piecewise polynomial function (e.g., a linear function $f\left(X_{B}\right)=a+b X_{B}$ where $a$ and $b$ are adjustable parameters);

3) Forwardly simulating the diffusion profile $C_{\text {sim }}(x)$ based on the first-guess $\widetilde{D}\left(X_{B}\right)$ values using the Fick's diffusion equations and assuming local equilibrium and mass balance at the phase interfaces for a multi-phase system.

4) Computing the total (summation of) least square errors between the simulated concentration profile $C_{\text {sim }}(x)$ and the smoothed experimental profile $C_{\text {expt }}(x)$ across the entire diffusion distance;

5) Repeatedly adjusting the parameters in the $\widetilde{D}\left(X_{B}\right)=\exp \left(f\left(X_{B}\right)\right)$ function and simulating the concentration profile $C_{\text {sim }}(x)$ until the summation of the least square errors between $C_{\text {sim }}(x)$ and $C_{\text {expt }}(x)$ reaches a minimum; and,

6) Accepting the final parameters that define the $\widetilde{D}\left(X_{B}\right)$ values across single-phase compositions as the extracted diffusion coefficients from the experimental profiles. 
The Darken's equation $\widetilde{D}=\left(X_{A} D_{B}^{*}+X_{B} D_{A}^{*}\right) \Phi$ relates the interdiffusion coefficient $\widetilde{D}$ to the mole fractions $X_{A}$ and $X_{B}$, the tracer diffusion coefficients $D_{A}^{*}$ and $D_{B}^{*}$ of A and B respectively, and the thermodynamic factor $\Phi$ (The vacancy wind factor is not included, but the effect is usually small) [23]. As the composition approaches pure elements, e.g., the mole fractions $X_{A}=1$ and $X_{B}=0$ (approaching pure $\mathrm{A}$ in this case), the thermodynamic factor $\Phi=1$, and $\widetilde{D}=D_{B}\left(X_{A}=1, X_{B}=0\right)$; thus the interdiffusion coefficient $\widetilde{D}$ equals the impurity diffusion coefficient of $\mathrm{B}$ in pure $\mathrm{A}, D_{B}$. Symmetrically, as the composition approaches pure $\mathrm{B}, \widetilde{D}=D_{A}\left(X_{A}=0, X_{B}=1\right)$, the interdiffusion coefficient $\widetilde{D}$ is identical to the impurity diffusivity of element $\mathrm{A}$ in pure B matrix, $D_{A}$. Therefore, the impurity diffusion coefficients can be obtained from the interdiffusion coefficients at the pure element concentrations using the forward-simulation analysis without the uncertainty of evaluating the slopes within the data scattering as compositions approach the pure elements.

Over the past three years, we employed the forward-simulation method to analyze dozens of diffusion concentration profiles obtained from diffusion couples and high-throughput diffusion multiples [24-26] that contain many diffusion couples in each sample. An example of a diffusion multiple is shown in Fig. 2 [27]. An elegant liquid-solid diffusion couple (LSDC) geometry recently developed by Zhong and Zhao [28] allows effective collection of diffusion profiles at temperatures above the eutectic point of a binary system by intentionally forming a liquid-phase pool inside a solid metal block. Forwardsimulation analysis of diffusion profiles from LSDCs has solved some of the most challenging cases of diffusion studies in low-eutectic systems as well as systems with extremely low solute solubilities [28]. A total of 133 impurity diffusion coefficient values were obtained from several sets of diffusion multiples and diffusion couples (Note: each value refers to an impurity diffusion coefficient of one solute in one pure element matrix at one temperature, not a dataset which contains three or more temperatures as discussed in the preceding paragraphs). Among them, 52 have tracer experimental values to be compared with, but one tracer value (Mo impurity diffusion in $\mathrm{Cr}$ ) was identified by Neumann and Tuijn [9] as problematic due to grain boundary diffusion and thus was not used for the comparison. Nineteen of the 52 values have two independent tracer measurements to be compared with, resulting in 71 comparison values that are shown in Fig. 3 and are listed in the Supplementary Information.

The horizontal axis in Fig. 3 is the literature tracer experimental data while the vertical axis is the experimental impurity diffusion coefficients obtained using forward-simulation analysis of experimental diffusion profiles. It should be noted that most of the tracer data are calculated using the Arrhenius equation with the activation energy $Q$ and the pre-factor $D_{0}$ provided in the literature and the temperature ranges are carefully checked to make sure only interpolated data and extrapolated data in very closeproximity to experimental data points are employed for the comparison.

Figure 3 shows an excellent agreement between the impurity diffusion coefficients obtained from the forward-simulation analysis on the experimental diffusion profiles of diffusion couples with those from tracer experiments reported in the literature. It provides direct validation for the capability of the forwardsimulation analysis in obtaining reliable impurity diffusion coefficients from diffusion couple profiles. To test the goodness of the agreement between our data and the tracer data from the literature, the standard deviation of $\log \left(\frac{D_{F-S}}{D_{\text {tracer }}}\right)$ is calculated for Fig. 3 and a T-test [29] for the data is also performed 
( $D_{F-S}$ is the impurity diffusion coefficients extracted using the forward-simulation analysis on experimental diffusion couple profiles and $D_{\text {tracer }}$ is the impurity diffusion coefficient data from the tracer experiments reported in the literature). For the 52 data points between forward-simulation results and 71 values from the tracer diffusion experiments in Fig. 3, the standard deviation for $\log \left(\frac{D_{F-S}}{D_{\text {tracer }}}\right)$ is 0.1904 , which indicates that the average deviation for the ratio $\frac{D_{F-S}}{D_{\text {tracer }}}$ is among $[0.65,1.55]$. The mean value of $\log \left(\frac{D_{F-S}}{D_{\text {tracer }}}\right)$ is 0.0418 , which leads to $\frac{D_{F-S}}{D_{\text {tracer }}}=1.101$. The statistical t-value for the T-test of these data is 1.852 , which is smaller than the critical value of the t-distribution $t_{0.025,71}=1.994$ [29], suggesting that the assumption that our impurity diffusion data are different from the tracer data reported in the literature is statistically invalid. In other words, the results suggest that there is no significant statistical difference between the impurity diffusion coefficients obtained using the forward-simulation analysis of experimental diffusion couple profiles and those from the tracer diffusion experiments.

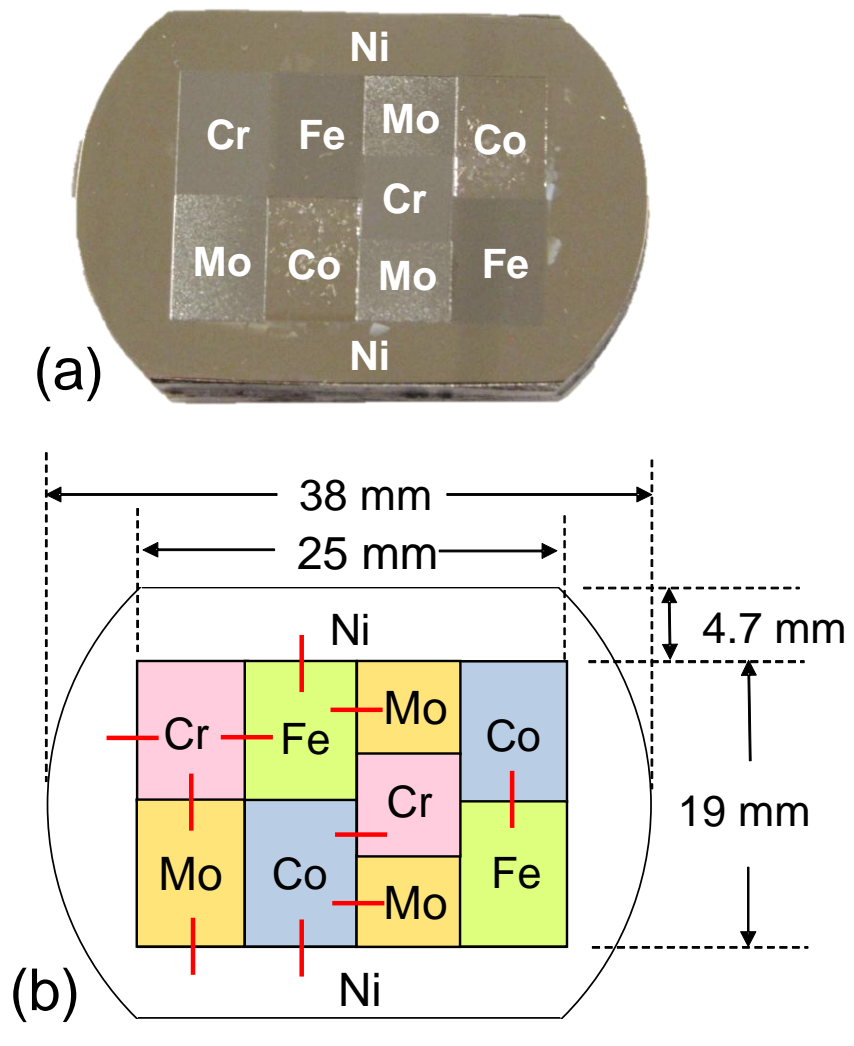

Fig. 2. A diffusion multiple example showing 10 binary diffusion couples included in one diffusion multiple as indicated by the short lines in (b)[27].

In conclusion, the forward-simulation analysis of experimental concentration profiles of regular (including pure element - pure element) diffusion couples can yield impurity diffusion coefficients as reliably and accurately as those obtained from the isotope tracer experiments. This new analysis together with high-throughput diffusion multiples that contain several diffusion couples in each sample provides a much more efficient way to obtain the much needed impurity diffusion coefficients that are the 
foundation of reliable diffusion (mobility) databases. The combined method is especially significant since thousands of impurity diffusion coefficient datasets are waiting to be determined and it is impractical to count on the extremely low number of tracer experiments to obtain those datasets.

\section{Acknowledgement}

This work was financially supported by the U.S. National Science Foundation (NSF) under award number NSF-DMR 1237577. The experimental concentration profiles were collected and analyzed under various funding sources including NSF-DMR 0804833, NSF-CMMI-1333999, and U.S. Department of Energy (DOE) DE-EE0006450. The forward-simulation program codes based on both MatLab and Python are available upon request from the authors. Data for Fig. 3 are tabulated in the Supplementary Information.

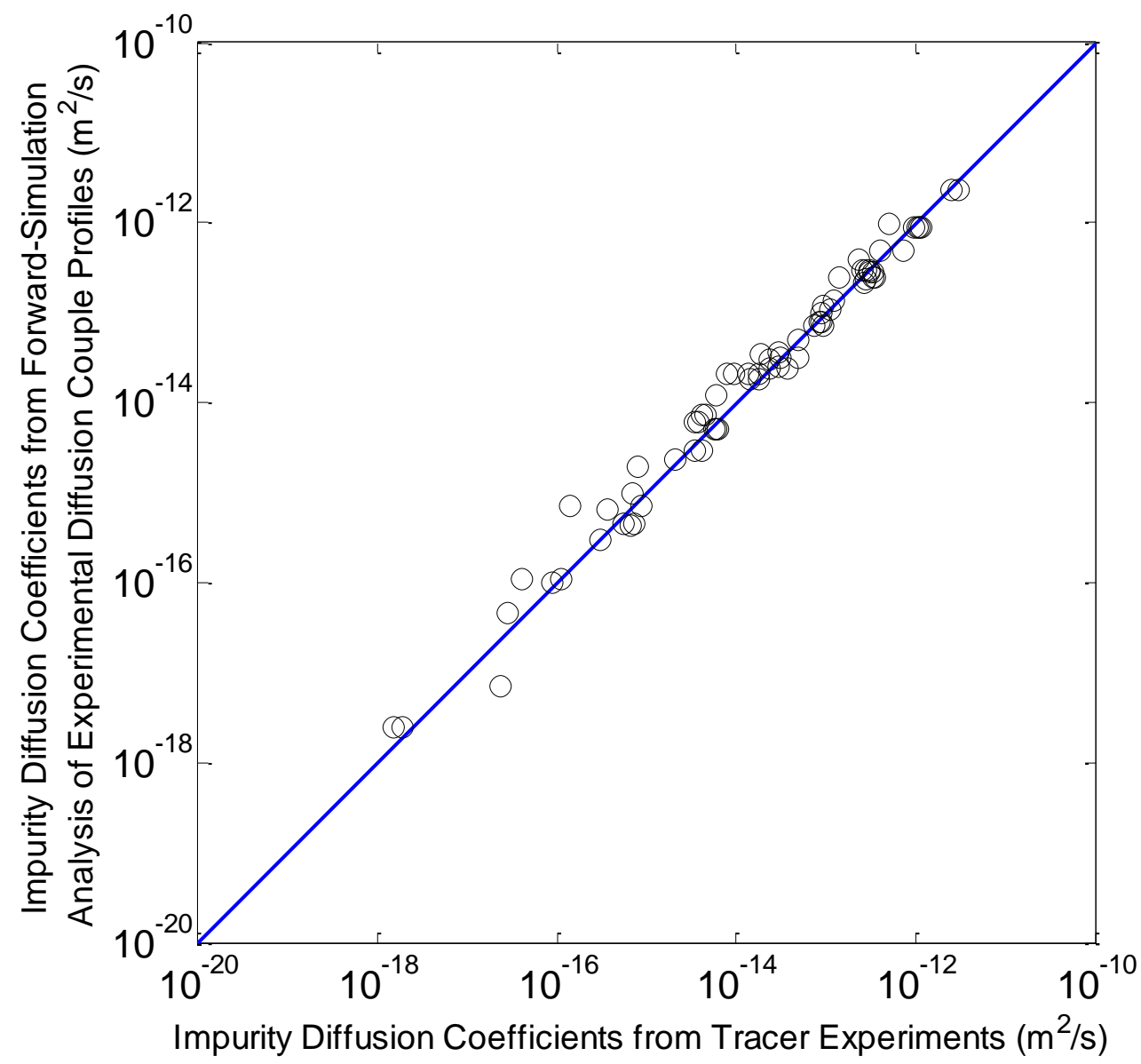

Fig. 3. Comparison of 52 impurity diffusion coefficients obtained from the forward-simulation analysis of experimental diffusion couple profiles with 71 isotope tracer experiment results reported in the literature (19 of the 52 have two independent tracer measurement values), showing an excellent agreement with no significant statistical difference; thus validating the reliability of the forward-simulation analysis in obtaining accurate impurity diffusion coefficients without using isotope tracer experiments. 


\section{References}

[1] White House Office of Science and Technology Policy (OSTP), Materials Genome Initiative: http://www.mgi.gov/ (Accessed in August 2016).

[2] T. Pollock, J. Allison, Integrated computational materials engineering: A transformational discipline for improved competitiveness and national security. Washington, DC: National Academies Press; 2008.

[3] J. Allison, D. Backman, L. Christodoulou, JOM 58 (2006) 25-27.

[4] J.-O. Andersson, J. Ågren, J. Appl. Phys. 72 (1992) 1350-1355.

[5] A.T. Dinsdale, CALPHAD 15 (1991) 317-425.

[6] L. Kaufman, E.V. Clougherty, R.J. Weiss, Acta Metall. 11 (1963) 323-335.

[7] H. Mehrer, Diffusion in solids: fundamentals, methods, materials, diffusion-controlled processes. Berlin: Springer-Verlag; 2007 (DOI: 10.1007/978-3-540-71488-0).

[8] K. Hirano, S. Fujikawa, J. Nucl. Mater. 69\&70 (1978) 564-566

[9] G. Neumann, C. Tuijn, editors. Self-diffusion and impurity diffusion in pure metals: handbook of experimental data. Amsterdam: Elsevier; 2009.

[10] S. Divinski, F. Hiskere, T. Wilger, M. Friesel, C. Herzig, Intermetallics 16 (2008) 148-155.

[11] J. Imai, O. Taguchi, G. Tiwari, Y. Iijima, Mater Trans JIM 55 (2014) 1786-1791.

[12] M. Mantina, Y. Wang, L.Q. Chen, Z.K. Liu, C. Wolverton, Acta Mater. 57 (2009) 4102-4108.

[13] H. Wu, T. Mayeshiba, D. Morgan, Sci. Data 3 (2016) 160054.

[14] B.-C. Zhou, S.-L. Shang, Y. Wang, Z.-K. Liu, Acta Mater. 103 (2016) 573-586.

[15] M. Badia, A. Vignes, Acta Metall. 17 (1969) 177-187.

[16] H. Araki, Y. Minamino, T. Yamane, T. Nakatsuka, Y. Miyamoto, Metall. Mater. Trans. A 27 (1996) 1807-1814.

[17] R.A. Perez, F. Dyment, H. Matzke, G. Linker, H. Dhers, J. Nucl. Mater. 217 (1994) 48-53.

[18] P.G. Shewmon, Diffusion in solids, 2nd Ed., New York: Wiley; 1991.

[19] F. Sauer, V. Freise, Zeit. Elektrochem. 66 (1962) 353-362.

[20] L. Hall, J. Chem. Phys. 21 (1953) 87-89.

[21] Q. Zhang, J.-C. Zhao, Intermetallics 34 (2013) 132-141.

[22] Q. Zhang, J.-C. Zhao, J. Alloys Compd. 604 (2014) 142-150.

[23] L.S. Darken, Trans. AIME 175 (1948) 184-294.

[24] J.-C. Zhao, Annu. Rev. Mater. Res. 35 (2005) 51-73.

[25] J.-C. Zhao, Prog. Mater. Sci. 51 (2006) 557-631.

[26] J.-C. Zhao, J. Mater. Sci. 39 (2004) 3913-3925.

[27] S. Cao, J.-C. Zhao, Acta Mater. 88 (2015) 196-206.

[28] W. Zhong, J.-C. Zhao, Scr. Mater. 127 (2017) 92-96.

[29] P.R. Nelson, K. Copeland, M. Coffin, Introductory statistics for engineering experimentation. New York: Academic Press; 2003. 


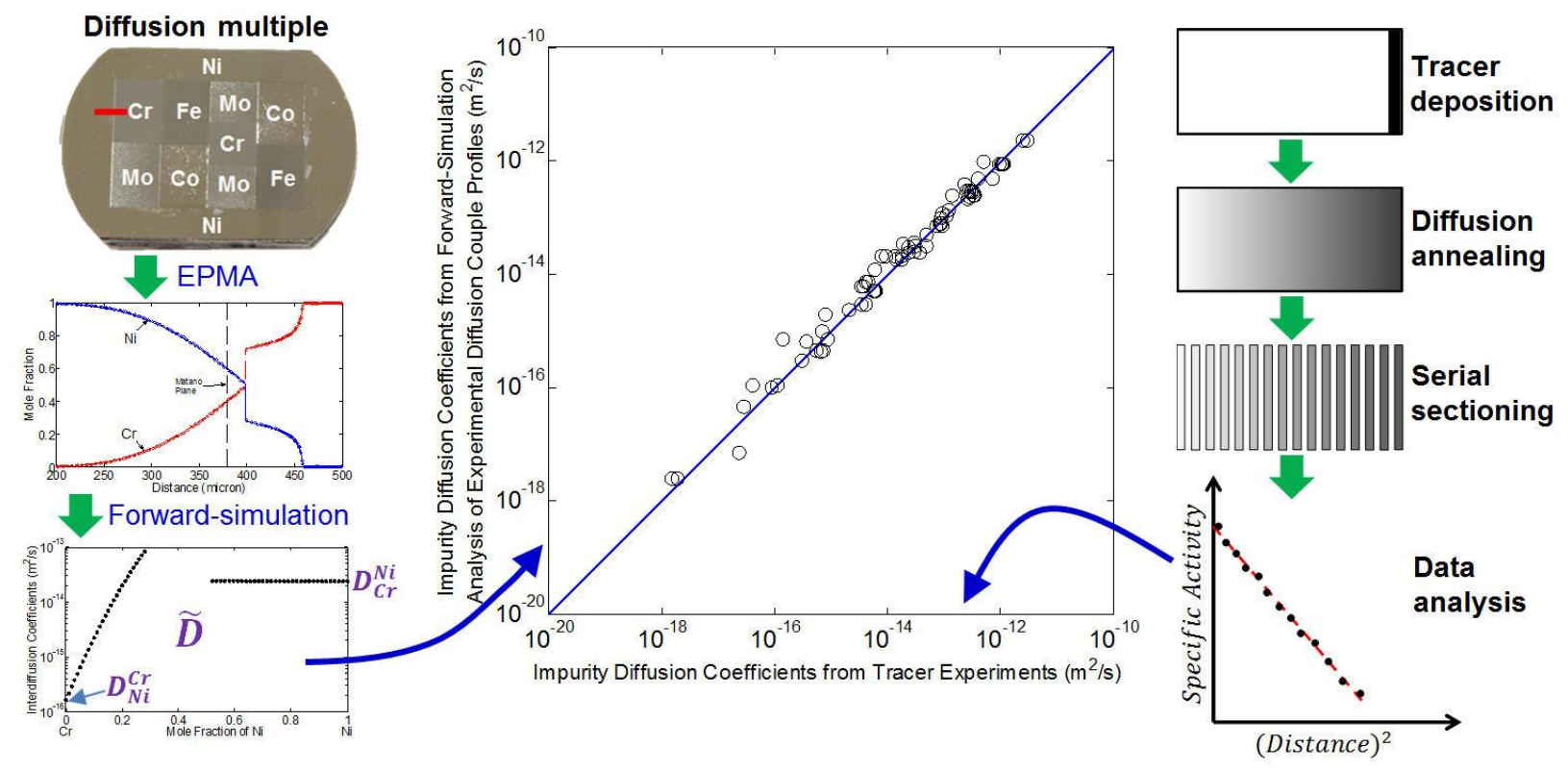

